\title{
Premature Valvular Heart Disease in Homozygous Familial Hypercholesterolemia
}

\author{
Akl C. Fahed, ${ }^{1}$ Kamel Shibbani, ${ }^{2}$ Rabih R. Andary, ${ }^{2}$ Mariam T. Arabi, ${ }^{2}$ \\ Robert H. Habib, ${ }^{3}$ Denis D. Nguyen, ${ }^{4}$ Fady F. Haddad, ${ }^{5}$ Elie Moubarak, \\ Georges Nemer, ${ }^{7}$ Sami T. Azar, ${ }^{8}$ and Fadi F. Bitar ${ }^{2,7}$ \\ ${ }^{1}$ Division of Cardiology, Massachusetts General Hospital, Boston, MA, USA \\ ${ }^{2}$ Departments of Pediatrics and Adolescent Medicine, American University of Beirut Medical Center, Beirut, Lebanon \\ ${ }^{3}$ The Society of Thoracic Surgeons, Chicago, IL, USA \\ ${ }^{4}$ Department of Gastroenterology, Hepatology, and Nutrition, Boston Children's Hospital, Boston, MA, USA \\ ${ }^{5}$ Surgery, American University of Beirut Medical Center, Beirut, Lebanon \\ ${ }^{6}$ National LDL Apheresis Center, Dahr El Bacheck Government University Hospital, Dahr El-Bachek, Lebanon \\ ${ }^{7}$ Biochemistry and Molecular Genetics, American University of Beirut Medical Center, Beirut, Lebanon \\ ${ }^{8}$ Internal Medicine, American University of Beirut Medical Center, Beirut, Lebanon
}

Correspondence should be addressed to Akl C. Fahed; fahed@mail.harvard.edu and Fadi F. Bitar; fbitar@aub.edu.lb

Received 20 January 2017; Revised 18 April 2017; Accepted 29 May 2017; Published 6 July 2017

Academic Editor: Maurizio Averna

Copyright (c) $2017 \mathrm{Akl} \mathrm{C.} \mathrm{Fahed} \mathrm{et} \mathrm{al.} \mathrm{This} \mathrm{is} \mathrm{an} \mathrm{open} \mathrm{access} \mathrm{article} \mathrm{distributed} \mathrm{under} \mathrm{the} \mathrm{Creative} \mathrm{Commons} \mathrm{Attribution} \mathrm{License,}$ which permits unrestricted use, distribution, and reproduction in any medium, provided the original work is properly cited.

Valvular heart disease frequently occurs as a consequence of premature atherosclerosis in individuals with familial hypercholesterolemia (FH). Studies have primarily focused on aortic valve calcification in heterozygous $\mathrm{FH}$, but there is paucity of data on the incidence of valvular disease in homozygous FH. We performed echocardiographic studies in 33 relatively young patients (mean age: 26 years) with homozygous FH (mean LDL of $447 \mathrm{mg} / \mathrm{dL}, 73 \%$ on LDL apheresis) to look for subclinical valvulopathy. Twentyone patients had evidence of valvulopathy of the aortic or mitral valves, while seven subjects showed notable mitral regurgitation. Older patients were more likely to have aortic valve calcification ( $>21$ versus $\leq 21$ years: $59 \%$ versus $12.5 \%$; $p=0.01$ ) despite lower LDL levels at the time of the study $(385$ versus $513 \mathrm{mg} / \mathrm{dL} ; p=0.016$ ). Patients with valvulopathy were older and had comparable LDL levels and a lower carotid intima-media thickness. Our data suggests that, in homozygous FH patients, valvulopathy (1) is present across a wide age spectrum and LDL levels and (2) is less likely to be influenced by lipid-lowering treatment. Echocardiographic studies that focused on aortic root thickening and stenosis and regurgitation are thus likely an effective modality for serial follow-up of subclinical valvular heart disease.

\section{Introduction}

In 1952, Dr. Barr and colleagues from the New York HospitalCornell Medical Center reported a case of a 20-year-old man with xanthomata who died from severe nonrheumatic aortic stenosis [1]. Autopsy of the heart showed extensive atherosclerosis in the aorta and aortic valve. This case report was long before the discovery of echocardiography, CT scanning, or the LDL receptor. Subsequent studies from the 1960s through the mid-1980s reported patients with xanthoma dying from valvular heart disease. These led to defining familial hypercholesterolemia as a disease characterized by accelerated atherosclerosis which could result in severe supravalvular aortic stenosis and eventually death. On autopsy, lipid deposition, calcification, and inflammation of the aorta were documented [2-4]. Since then, scientific discovery has led to the cloning of the LDL receptor, development of lipid-lowering therapy, and improvement of diagnosis of valvular heart disease with echocardiography.

Familial hypercholesterolemia (FH) is a Mendelian disorder of abnormally high low-density lipoprotein (LDL) cholesterol. In the homozygous or severe form which occurs 
in a prevalence rate of around 1 in a million, LDL levels usually exceed 3 to 4 times the upper limit of normal. FH patients often present with external cholesterol depositions around their eyes (xanthelasmas) or joints (xanthomas) and are particularly prone to premature atherosclerotic cardiovascular disease (CVD) [5]. FH patients often require treatment with plasmapheresis in addition to lipid-lowering medications.

Clinical prognosis in $\mathrm{FH}$ focuses on prevalence of atherosclerotic CVD and its surrogate markers. However, a lesser studied impact of the disorder is the increased risk of developing valvular heart disease. Hypercholesterolemic valvulopathy remains a vague entity in FH patients [6]. Calcific aortic valve disease is the most described, with sparing literature detailing early mitral valve abnormalities $[1,2]$. Multiple studies suggested that aortic valve calcification, best seen on computer tomography (CT) scan, is the earliest maker of aortic valve disease in $\mathrm{FH}$ patients $[7,8]$. However, aortic valve calcification does not correlate with LDL levels or treatment in $\mathrm{FH}$ patients, and multiple randomized control clinical trials have shown no improvement of aortic valve calcification with lipid-lowering therapy [9-11]. There have been two cohort studies that looked at 10 and 39 homozygous $\mathrm{FH}$ patients, respectively $[12,13]$. While patients in these studies had aortic valve calcification, there was high prevalence of aortic stenosis and regurgitation. Kawaguchi et al. studied 10 homozygous patients and 8 of them had aortic valve stenosis or regurgitation [12]. Kolansky et al. found that 8 out of 39 homozygous FH patients (21\%) had mild-to-moderate aortic regurgitation [13]. The same study showed in longitudinal follow-up of a small group of the cohort that aortic regurgitation might be the earliest manifestation of valvulopathy in these patients and is associated with progression to clinically significant CVD [13]. These studies were limited by the small sample size and the heterogeneity of patients and testing done. The exact prevalence and distribution of subclinical valvular disease in this population remain unknown. No study has reported the complete echocardiographic findings on a sizeable cohort of relatively young, well phenotyped and genotyped, homozygous FH patients without previously known significant CVD.

We have previously reported genetic studies on the Lebanese FH Registry, which is one of the largest reported cohorts of homozygous FH in the literature [14, 15]. In this study, the same cohort of 33 patients is interrogated for evidence of subclinical premature valvular heart disease using echocardiography. We aimed to define the prevalence and characteristics of premature subclinical valvular heart disease in patients with severe $\mathrm{FH}$ using echocardiography and determine how factors such as LDL levels, plasmapheresis treatment, genotype, and age affect the disease.

\section{Methods}

2.1. Patients and Phenotypes. The study was approved by the Institutional Review Board of the American University of Beirut, and all patients or parents gave informed consent. Thirty-three Lebanese patients (mean age: $26 ; 51 \%$ female) with severe (clinically homozygous) hypercholesterolemia were recruited to the study from the Lebanese FH Registry as previously described. A detailed medical history, physical exam, and review of the medical records were performed by two clinicians. Fasting lipid panel measurements were obtained. This was drawn prior to an LDL apheresis session for those patients receiving this treatment. Genetic information on all subjects was also available from a prior study [14].

2.2. Cardiovascular Evaluations. Routine $2 \mathrm{D}$ echocardiography with color and Doppler was performed on all subjects in the study by two experienced cardiologists in echocardiography who were blinded to the clinical history of the patients. Two patients (67 and 76) had echocardiograms performed at a different institution and were reviewed by the same cardiologists. Valvulopathy was defined as aortic valve calcification, aortic root thickening, and any valvular stenosis or regurgitation that is mild or more. Aortic root thickening was defined as an anterior or posterior wall thickness of $>2.2 \mathrm{~mm}$ as measured by $\mathrm{M}$-mode at the end diastolic dimension of the aortic root walls at the sinuses and tubular portions from the longitudinal or short axis view. Aortic stenosis was defined as follows: mild (aortic jet velocity of $2.0-2.9 \mathrm{~m} / \mathrm{s}$ and a peak systolic gradient and a mean gradient of $<36 \mathrm{mmHg}$ and $<20 \mathrm{mmHg}$, resp.), moderate (aortic jet velocity of $3.0-4.0 \mathrm{~m} / \mathrm{s}$ and a peak systolic gradient and a mean gradient of $36-64 \mathrm{mmHg}$ and $20-40 \mathrm{mmHg}$, resp.), and severe (aortic jet velocity of $>4 \mathrm{~m} / \mathrm{s}$ and a peak systolic gradient and a mean gradient of $>64 \mathrm{mmHg}$ and $>40 \mathrm{mmHg}$, resp.). Carotid duplex ultrasound and measurement of intimamedia thickness were performed as previously described [16]. Ankle-brachial index (ABI) was measured for all patients on the right and left dorsalis pedis and posterior tibialis. Any ABI of $\leq 0.90$ was considered abnormal based on the most recent ACC/AHA guidelines [17]. Presence of prior cardiovascular disease was defined as coronary artery disease requiring revascularization, nonembolic stroke, or carotid stenosis requiring surgery.

2.3. Statistical Analyses. Two group comparisons for summary measures were performed. Fisher's exact test was used to compare categorical variables. Continuous variables were not normally distributed and were compared using Wilcoxon's rank-sum test. A $p$ value less than or equal to 0.05 was considered statistically significant. All analyses were performed using Stata/SE v.12.0 (Stata Corp., College Station, Texas).

\section{Results}

Thirty-three patients (52.5\% female, mean age: 26 years) were studied. Patients in our cohort tended to have a rather severe phenotypic presentation as demonstrated by a mean LDL of $447 \mathrm{mg} / \mathrm{dL}$ and the fact that $73 \%$ were on plasmapheresis. Genetically, $82 \%$ had homozygous or compound heterozygous mutations in the $L D L R$ or $A R H$ genes, and the remainder had heterozygous mutations in these genes. Only 7 patients (21\%, average age: 49 years) had known atherosclerotic CVD prior to enrollment in the study (Tables 1,2, and 3). Subclinical vascular disease was also present with mean (average of right and left for all patients) carotid intima-media 
TABLE 1: Demographic and clinical characteristics.

\begin{tabular}{|c|c|c|c|c|}
\hline Characteristics & All patients $(N=33)$ & $\leq 21(N=16)$ & $>21(N=17)$ & $p$ value \\
\hline Age, mean (SD) & $26.12(16.40)$ & $13.56(4.97)$ & $37.94(14.45)$ & \\
\hline Female, \% $(N)$ & $51.5(17)$ & $68.75(11)$ & $35.29(6)$ & 0.084 \\
\hline LDL (mg/dL), mean (SD) & $447.45(151.2)$ & $513.40(114)$ & $385.30(158.31)$ & 0.016 \\
\hline HDL (mg/dL), mean (SD) & $45.43(26.88)$ & $46.94(33.26)$ & $44.02(20.10)$ & 0.87 \\
\hline Genetically homozygous, $\%(N)$ & $57.58(19)$ & $68.75(11)$ & $47.06(8)$ & 0.30 \\
\hline Apheresis, \% (N) & $72.73(24)$ & $62.50(10)$ & $82.35(14)$ & 0.26 \\
\hline Xanthoma, \% (N) & $93.94(31)$ & $93.75(15)$ & $94.12(16)$ & 1 \\
\hline Xanthelasma, \% $(N)$ & $39.39(13)$ & $25(4)$ & $52.94(9)$ & 0.16 \\
\hline Arcus senilis, \% (N) & $42.42(14)$ & $37.50(6)$ & $47.60(8)$ & 0.73 \\
\hline IMT, mean (SD) & $0.87(0.57)$ & $0.67(0.45)$ & $1.06(0.62)$ & 0.084 \\
\hline Reduced $\mathrm{ABI}^{\alpha}, \%(N)$ & $18.18(6)$ & $6.25(1)$ & $29.41(5)$ & 0.17 \\
\hline Clinical CVD, \% (N) & $21.21(7)$ & $0(0)$ & $41.18(7)$ & 0.0072 \\
\hline Valvulopathy $^{\beta}, \%(N)$ & $63.6(21)$ & $33.33(7)$ & $82.35(14)$ & 0.032 \\
\hline Aortic valve disease, $\%(N)$ & $63.63(21)$ & $43.75(7)$ & $82.35(14)$ & 0.032 \\
\hline Calcification, \% $(N)$ & $36.36(12)$ & $12.50(2)$ & $58.82(10)$ & 0.01 \\
\hline Root thickening, \% (N) & $42.42(14)$ & $25(4)$ & $58.82(10)$ & 0.08 \\
\hline Regurgitation, \% (N) & $24.24(8)$ & $12.50(2)$ & $35.29(6)$ & 0.22 \\
\hline Stenosis, \% $(N)$ & $39.39(13)$ & $37.50(6)$ & $41.18(7)$ & 1 \\
\hline Mitral valve regurgitation, $\%(N)$ & $21.21(7)$ & $12.50(2)$ & $23.53(4)$ & 0.66 \\
\hline
\end{tabular}

LDL: low-density lipoprotein cholesterol; HDL: high-density lipoprotein; ABI: ankle-brachial index; IMT: intima-media thickness; CVD: cardiovascular disease. ${ }^{\alpha}$ Reduced ABI was defined as any ABI (among right or left dorsalis pedis or posterior tibialis) that is $\leq 0.9$. ${ }^{\beta}$ Valvulopathy includes patients with calcifications/aortic root thickening only $(N=4,12.1 \%$ of the cohort) or valvular stenosis or regurgitation with or without calcifications $(N=17,51.5 \%$ of the cohort).

thickness (IMT) of $0.87 \mathrm{~mm}$ and $18 \%$ of patients having a reduced ankle-brachial index (ABI) (Table 1).

Despite the young age of the cohort, $64 \%$ (21 subjects) had subclinical valvular heart disease on echocardiography (Table 1 and Figure 1). Five out of 7 patients with known atherosclerotic CVD had also valvular findings on echocardiography and two did not. Excluding the 7 patients with known atherosclerotic CVD, 61.5\% (16 out of 26) had subclinical valvular heart disease on echocardiography. All valvular pathology involved the left-sided heart valves. Of the 21 subjects with valvulopathy, all had one or more aortic pathologies, while 6 had concomitant mitral regurgitation (Figure 1). Among the aortic valve pathologies, root thickening was the most common (14 out of 21), followed by aortic valve stenosis (13 out of 21) and aortic valve calcification (12 out of 21) (Table 1). All patients with aortic stenosis (AS) had valvular aortic stenosis. These included three patients with moderate AS and ten patients with mild AS. Nine patients ( $43 \%$ of valvulopathy patients) had aortic root thickening or valve regurgitation or stenosis in the absence of aortic valve calcification, and three patients (14, 6, and 17) had calcification without other valvular or root pathologies (Table 3). These patients were distributed also across a wide age spectrum.

The cohort had a median age of 24 years. The younger half of the cohort ( $\leq 21$ years) had significantly higher mean LDL levels compared to the older half ( $>21$ years). All seven patients with known cardiovascular disease were also $>21$ years old. The older patients were also more likely to have

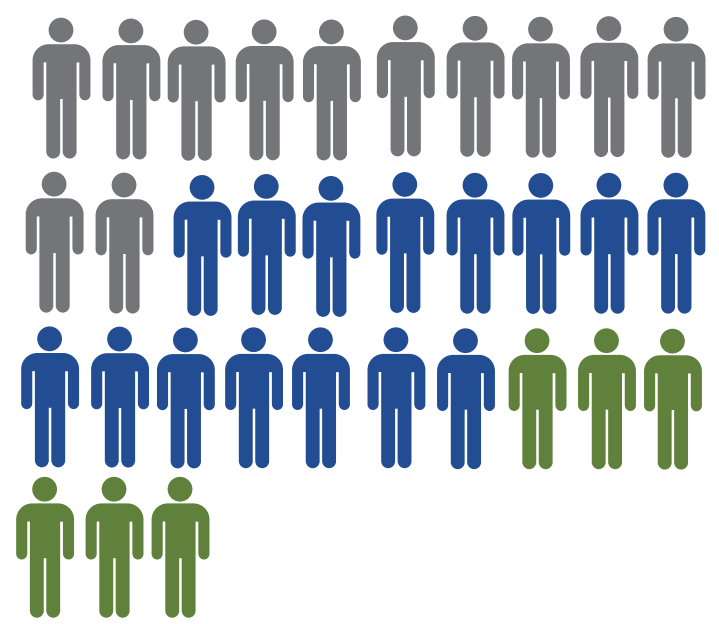

12, no valvulopathy 15 , aortic valvulopathy 6 , aortic and mitral valvulopathy

Figure 1: Prevalence of FH valvulopathy in homozygous FH. A diagram of the homozygous $\mathrm{FH}$ cohort showing patients without valvulopathy (grey), with aortic valvulopathy (blue), and with both aortic and mitral valvulopathies (green).

valvular heart disease (82\%) as compared to $33 \%$ in the younger patients $(p=0.032)$. The difference was also statistically significant for aortic valve calcification $(58 \%$ versus $12 \%, p=0.01$ ) (Table 1 ). 
TABLE 2: FH patients with and without valvulopathy.

\begin{tabular}{lccc}
\hline Characteristics & Valvulopathy $(N=21)$ & No valvulopathy $(N=12)$ & $p$ value \\
\hline Age, mean $( \pm \mathrm{SD})$ & $31(14.41)$ & $17.5(16.66)$ & $50(6)$ \\
Female, $\%(N)$ & $52.38(11)$ & $466.25(149.21)$ & 0.020 \\
LDL $(\mathrm{mg} / \mathrm{dL})$, mean $( \pm \mathrm{SD})$ & $436.7(154.85)$ & $55.89(36.29)$ & 0.50 \\
HDL $(\mathrm{mg} / \mathrm{dL})$, mean $( \pm \mathrm{SD})$ & $39.46(18.14)$ & $75(9)$ & 0.13 \\
Genetically homozygous, $\%(N)$ & $85(18)$ & $50(6)$ & 0.64 \\
Apheresis, $\%(N)$ & $85.7(18)$ & $3.92(5.32)$ & 0.044 \\
Apheresis years, mean $( \pm \mathrm{SD})$ & $7.33(4.46)$ & $1.01(0.64)$ & 0.072 \\
IMT, mean $( \pm \mathrm{SD})$ & $0.62(0.31)$ & $16.67(2)$ & 0.035 \\
Reduced ABI, $\%(N)$ & $28.57(6)$ & $33.33(4)$ & 0.68 \\
Clinical CVD, $\%(N)$ & $38.10(8)$ & & 1 \\
\hline
\end{tabular}

Patients with valvulopathy $(n=21)$ were on average older than those without valvulopathy $(n=12)$ (31 versus 17 years, $p=0.02)$ and were more likely to be on apheresis $(85 \%$ on apheresis for an average duration of 7 years versus $50 \%$ for an average of 4 years) (Table 2). Table 3 shows the patients ranked by increasing age. Older patients tend to have more diffuse disease involving the aortic valve, aortic root, mitral valves, and carotid. Finally, patients with valvular disease had a mean IMT $(0.62 \mathrm{~mm})$ that was smaller than that of those without $(1.01 \mathrm{~mm}, p=0.035)$ (Table 2).

\section{Discussion}

In this cohort study of 33 relatively young patients with severe $\mathrm{FH}$, the major findings were as follows: (1) subclinical $\mathrm{FH}$ valvulopathy was present in $64 \%$ of patients on echocardiography; (2) aortic root thickening, aortic stenosis, aortic regurgitation, and mitral regurgitation occurred commonly in severe $\mathrm{FH}$ patients and sometimes in the absence of aortic valve calcification; (3) older patients were more likely to have aortic valve calcification despite lower LDL-C levels; and (4) patients with valvulopathy were older and were more likely to be on LDL apheresis but with lower intima-media thickness compared to patients who did not have valvulopathy.

The findings from this study expand our understanding of the subclinical premature occurrence of valvular heart disease in severe FH. Besides several case reports, there have only been two published echocardiography studies of 39 and 10 severe FH patients, respectively $[12,13]$. Both studies highlighted $\mathrm{FH}$ valvulopathy as a distinct feature from aortic valve calcification seen in heterozygous $\mathrm{FH}$ patients, often using computed tomography [7]. FH valvulopathy is characterized by involvement of the left-sided valves, primarily the aortic valve. Kolansky et al. concluded that aortic regurgitation is the most prevalent and earliest sign of $\mathrm{FH}$ valvulopathy, and it was associated with presence of angiographic coronary stenosis [13]. In a smaller cohort, Kawaguchi et al. reported that 8 out of the 10 homozygous $\mathrm{FH}$ patients had aortic valve stenosis [12]. No significant involvement of mitral valve disease in homozygous $\mathrm{FH}$ patients was reported in the two major cohorts cited or prior case reports. However, in a group of 39 heterozygous $\mathrm{FH}$ patients, the prevalence of mitral regurgitation was $41 \%$ but was not significantly different from a control group [12]. In our study, we confirm previous findings that, in severe $\mathrm{FH}$, valvulopathy on echocardiography involved regurgitant and stenotic aortic valvular phenotypes without necessarily calcification. In contrast to the prior two cohorts, we show that valvulopathy phenotype is heterogeneous and is not limited to aortic regurgitation or stenosis. In fact, the single most common finding was aortic root thickening in $42 \%$ of patients $(n=14)$, while mitral regurgitation was seen in $21 \%$ of patients $(n=6)$. Overall, $64 \%$ of patients $(n=18)$ had at least one abnormal valve finding on echocardiography. All six patients who had mitral regurgitation also had aortic valvulopathy.

Most of the studies investigating valvular heart disease in $\mathrm{FH}$ have focused on aortic valve calcification. In one study, cardiac CT was used to screen a large cohort of older heterozygous $\mathrm{FH}$ patients for aortic valve calcification and found that it occurred in $41 \%$ of cases compared to $21 \%$ of controls $(p<0.001)$ [7]. In that study, aortic valve calcification was independently associated with age, LDL levels, and diastolic blood pressure and was predicted strongly by the presence of a loss of function mutation in the LDL receptor gene (OR: 4.81, $p<0.001$ ) [7]. Other studies reported prevalence of aortic valve calcification approaching $100 \%$ in homozygous $\mathrm{FH}[18,19]$. In this study, $43 \%$ of patients with valvulopathy did not have aortic valve calcification and three had aortic valve calcification without evidence of other pathologies on echocardiography. Echocardiogram is less sensitive than cardiac CT scan in detecting aortic valve calcification and as such it is possible that some of the patients in our study have calcifications that are not detected on echocardiography.

Aortic stenosis in severe FH was one of the first cardiovascular phenotypes to be described in several case reports prior to echocardiography based on clinical exam, hemodynamic assessment, and autopsy [1-4]. These severe presentations, in an era prior to LDL apheresis or statins, were a good depiction of the natural history of disease. Severe supravalvular aortic stenosis continues to be seen on echocardiography on presentation of untreated severe FH children [20]. In an older study form 1989 describing 18 patients with homozygous $\mathrm{FH}$, supravalvular stenosis was also common, detected in $23 \%(3 / 13)$ of patients by echocardiography and 54\% (6/11) of the cohort by catheterization [21]. With LDL-lowering 


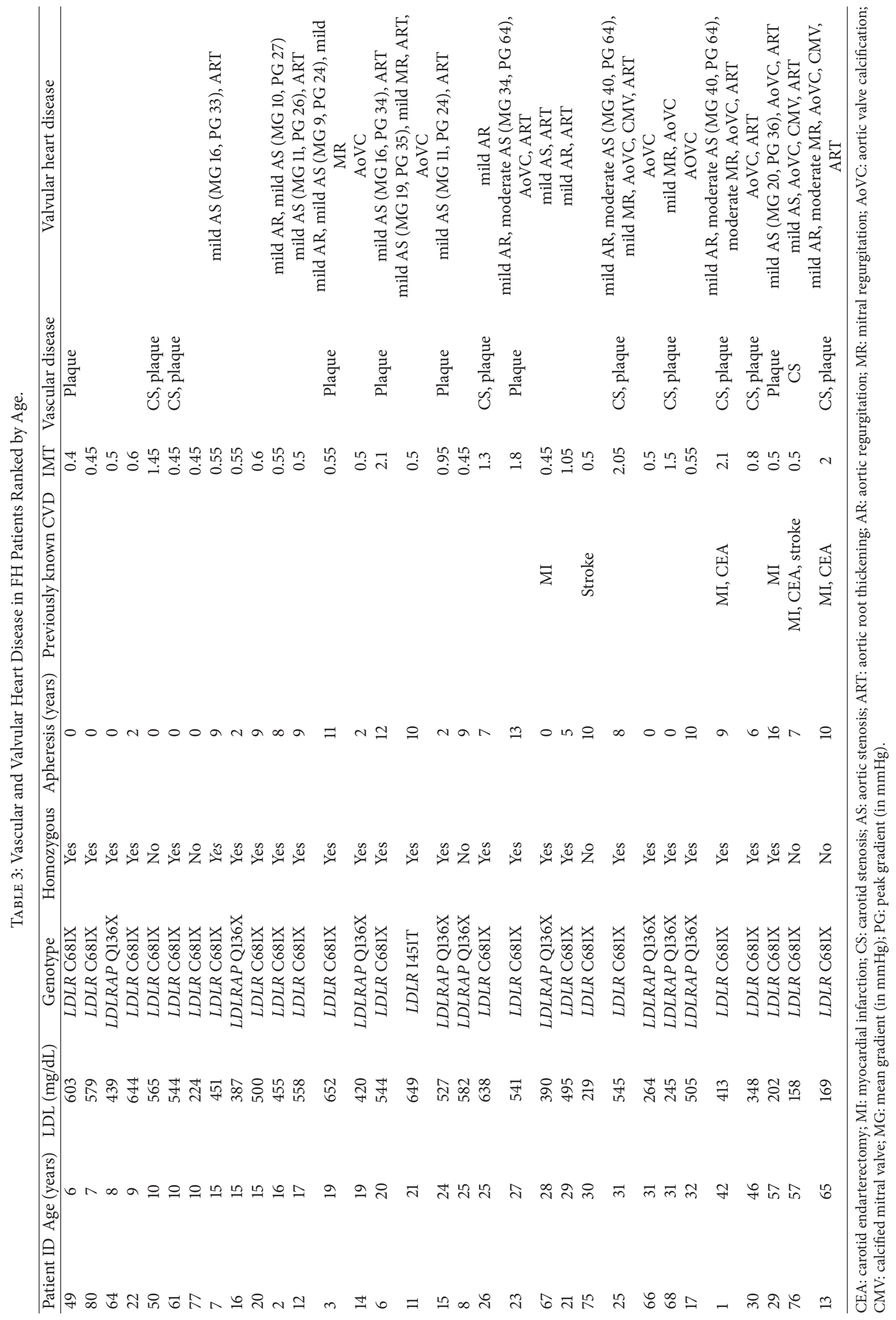


with both lipid-lowering therapy and LDL apheresis, the severity of AS is less but is still higher than what you would expect in otherwise healthy young population $[12,13]$. In our cohort, 13 patients (40\%) had aortic stenoses which were all mild with the exception of patient 1 ( 42 years old) who had moderate AS. He also has severe carotid stenosis and coronary artery disease. Although all aortic stenoses were valvular, it is likely that aortic root thickening reflects a milder form of supravalvular cholesterol deposition. The patients with aortic stenosis were distributed across wide age spectrum, LDL levels, and duration of lipid-lowering therapy. The low sample size does not allow making conclusions regarding specific characteristics of those patients with AS. Similarly, 8 patients (24\%) had aortic regurgitation, five of which also had concomitant stenosis. This prevalence is lower than the one reported by Kolansky et al. [13]. Relying on aortic regurgitation as the only earliest indicator of valvular pathology in this population would likely miss a large portion of $\mathrm{FH}$ patients with subclinical valvular heart disease.

Aortic root thickening was the most prevalent finding in our patients (42\%) and often coincided with not only aortic regurgitation but also calcified valve and aortic stenosis. Root thickening per se on echocardiography had not been described in the two prior cohorts. Kawaguchi described cusp thickening as a result of lipid accumulation as well as infiltration of inflammatory cells, which eventually leads to regurgitation [6]. We believe that a similar process occurs in the aortic root and is likely the earliest manifestation of supravalvular aortic stenosis which we see in our study but it also has been well described in the multiple untreated case reports in the early literature $[3,4,20,22]$. Also atheromatous plaques in the root and ascending aorta have been described in heterozygous FH patients with lower LDL levels [12]. Additionally, the internal diameter of the supravalvular aortic ridge has been shown to be smaller in FH patients compared to controls, with reduced distensibility [12]. The hemodynamics of the aortic root have been further studied, showing that FH patients have increased aortic stiffness and reduced distensibility [23].

Mitral valvulopathy is less described in $\mathrm{FH}$ patients. Eight patients (24\%) had mitral regurgitation in our cohort of severe FH patients. These included at least three young patients (patient IDs 3, 11, and 68 with ages of 19, 21, and 31 years, resp.) without a history of moderate or above AS or known coronary artery disease to justify ischemia or cardiomyopathy as a cause of the mitral regurgitation. While mitral regurgitation in this population could occur secondary to ischemia and cardiomyopathy, our data do suggest a primary valvulopathy of the mitral valve. We hypothesize the three potential mechanisms, silent subclinical ischemia of the papillary muscles, calcification of the mitral annulus, or hemodynamic strain on the left ventricle, as a consequence of the decreased distensibility of the aorta.

Limited studies suggested the presence of molecular mechanisms of FH valvulopathy independent of LDL Levels but related to other downstream effects of the genetic mutation [8]. We are unable, however, in our case to see any difference between patients with homozygous (complete loss of LDL receptor function) and nonhomozygous mutations. This could be limited by the small power of our study to detect such differences which will always be challenging with severe $\mathrm{FH}$ given the low prevalence of disease. This is consistent with our prior findings that LDL levels and not mutation status predict carotid intima-media thickness in FH [16]. However, patients with valvulopathy, despite being older and having comparable LDL levels than those without valvulopathy, had a significantly lower intima-media thickness. This suggests that valvulopathy, unlike intima-media thickness, is less likely to correlate with fluctuations in LDL levels, that is, reduction of intima-media thickness without resolution of valvular findings after getting apheresis. This remains, however, to be proven by longitudinal studies. It is possible that patients with FH are exposed to high LDL very early on in life, and this exposure is what triggers downstream molecular pathways leading to irreversible structural valvular changes. A factor of LDL levels multiplied by age or duration of exposure (area under the curve) seems to be the major determinant of valvulopathy.

This study has multiple limitations. First it is a crosssectional study and as such using age and duration of treatment can only suggest trends in progression of disease; conclusions regarding progression of disease could only be made with longitudinal follow-up. Second, the study did not look specifically at calcifications using cardiac CT to determine how this correlated with echocardiographic findings. Finally, lipoprotein-a (LPA) levels were not measured as part of the study. Recent studies have shown that genetically determined elevation in LPA levels is associated with aortic stenosis in the general population [24] and is also an independent risk factor of aortic valve calcification in $\mathrm{FH}$ [25].

Findings of very high prevalence of subclinical valvular heart disease in a young population of severe $\mathrm{FH}$ patients have important clinical implications on the management of these patients. While this study is cross-sectional, we know from the natural history of disease that severe symptomatic supravalvular aortic stenosis occurs in untreated patients. Also limited longitudinal data has shown that at least subclinical aortic regurgitation correlates with clinically significant cardiovascular disease [13]. Based on this, it is reasonable to recommend echocardiography at diagnosis and serially every 1-2 years on all patients with this severe $\mathrm{FH}$.

\section{Conclusion}

We show that subclinical valvular heart disease could be detected in a very high proportion of severe $\mathrm{FH}$ patients using echocardiography. We also characterize $\mathrm{FH}$ valvulopathy as a combination of one or more of aortic root thickening, aortic valve regurgitation, stenosis or calcification, and mitral regurgitation which progresses with age on treatment but at a remarkably accelerated rate.

\section{Conflicts of Interest}

The authors declare that they have no conflicts of interest. 


\section{Authors' Contributions}

Akl C. Fahed and Kamel Shibbani contributed equally to this work.

\section{Acknowledgments}

The authors would like to thank the patients and their families for participation in the study. The study was funded by a grant from the Lebanese National Center for Scientific Research.

\section{References}

[1] D. P. Barr, S. Rothbard, and H. A. Eder, "Atherosclerosis and aortic stenosis in hypercholesteremic xanthomatosis," Journal of the American Medical Association, vol. 156, no. 10, pp. 943-947, 1954.

[2] S. Rothbard, J. W. C. Hagstrom, and J. P. Smith, "Aortic stenosis and myocardial infarction in hypercholesterolemic xanthomatosis," American Heart Journal, vol. 73, no. 5, pp. 687692, 1967.

[3] A. Wennevold and J. G. Jacobsen, "Acquired supravalvular aortic stenosis in familial hypercholesterolemia. A hemodynamic and angiocardiographic study," The American Journal of Medicine, vol. 50, no. 6, pp. 823-827, 1971.

[4] D. L. Sprecher, E. J. Schaefer, K. M. Kent et al., "Cardiovascular features of homozygous familial hypercholesterolemia: analysis of 16 patients," The American Journal of Cardiology, vol. 54, no. 1, pp. 20-30, 1984.

[5] A. C. Fahed and G. M. Nemer, "Familial hypercholesterolemia: The lipids or the genes?" Nutrition and Metabolism, vol. 8, article no. 23, 2011.

[6] A. Kawaguchi, C. Yutanid, and A. Yamamoto, "Hypercholesterolemic valvulopathy: An aspect of malignant atherosclerosis," Therapeutic Apheresis, vol. 7, no. 4, pp. 439-443, 2003.

[7] G.-J. R. T. Kate, S. Bos, A. Dedic et al., "Increased Aortic Valve Calcification in Familial Hypercholesterolemia Prevalence, Extent, and Associated Risk Factors," Journal of the American College of Cardiology, vol. 66, no. 24, pp. 2687-2695, 2015.

[8] K. Alrasadi, K. Alwaili, Z. Awan, D. Valenti, P. Couture, and J. Genest, "Aortic calcifications in familial hypercholesterolemia: Potential role of the low-density lipoprotein receptor gene," American Heart Journal, vol. 157, no. 1, pp. 170-176, 2009.

[9] S. J. Cowell, D. E. Newby, R. J. Prescott et al., "A randomized trial of intensive lipid-lowering therapy in calcific aortic stenosis," New England Journal of Medicine, vol. 352, no. 23, pp. 2389-2397, 2005.

[10] A. B. Rossebo, T. R. Pedersen, and K. Boman, "Intensive lipid lowering with simvastatin and ezetimibe in aortic stenosis," The New England Journal of Medicine, vol. 359, pp. 1343-1356, 2008.

[11] K. L. Chan, K. Teo, J. G. Dumesnil, A. Ni, and J. Tam, "Effect of lipid lowering with rosuvastatin on progression of aortic stenosis: results of the aortic stenosis progression observation: measuring effects of rosuvastatin (ASTRONOMER) trial," Circulation, vol. 121, no. 2, pp. 306-314, 2010.

[12] A. Kawaguchi, K. Miyatake, C. Yutani et al., "Characteristic cardiovascular manifestation in homozygous and heterozygous familial hypercholesterolemia," American Heart Journal, vol. 137, no. 3, pp. 410-418, 1999.

[13] D. M. Kolansky, M. Cuchel, B. J. Clark et al., "Longitudinal Evaluation and Assessment of Cardiovascular Disease in Patients
With Homozygous Familial Hypercholesterolemia," American Journal of Cardiology, vol. 102, no. 11, pp. 1438-1443, 2008.

[14] A. C. Fahed, R. Khalaf, R. Salloum et al., "Variable expressivity and co-occurrence of LDLR and LDLRAP1 mutations in familial hypercholesterolemia: failure of the dominant and recessive dichotomy," Molecular Genetics \& Genomic Medicine, vol. 4, no. 3, pp. 283-291, 2016.

[15] A. C. Fahed, R. M. Safa, F. F. Haddad et al., "Homozygous familial hypercholesterolemia in Lebanon: a genotype/phenotype correlation," Molecular Genetics and Metabolism, vol. 102, no. 2, pp. 181-188, 2011.

[16] A. C. Fahed, R. H. Habib, G. M. Nemer et al., "Low-density lipoprotein levels and not mutation status predict Intima-media thickness in familial hypercholesterolemia," Annals of Vascular Surgery, vol. 28, no. 2, pp. 421-426, 2014.

[17] T. W. Rooke, A. T. Hirsch, S. Misra et al., "ACCF/AHA Focused Update of the Guideline for the Management of Patients With Peripheral Artery Disease (updating the 2005 guideline): a report of the American College of Cardiology Foundation/ American Heart Association Task Force on Practice Guidelines," Journal of the American College of Cardiology, vol. 58, no. 18, pp. 2020-2045, 2011.

[18] Z. Awan, K. Alrasadi, G. Francis et al., "Vascular Calcifications in Homozygote Familial Hypercholesterolemia," Arteriosclerosis, Thrombosis, and Vascular Biology, vol. 28, no. 4, pp. 777-785, 2008.

[19] J. M. Hoeg, I. M. Feuerstein, and E. E. Tucker, "Detection and quantitation of calcific atherosclerosis by ultrafast computed tomography in children and young adults with homozygous familial hypercholesterolemia," Arteriosclerosis, Thrombosis, and Vascular Biology, vol. 14, no. 7, pp. 1066-1074, 1994.

[20] S. Rafeiyian, S. Mojtahedzadeh, M. Hekmat et al., "Supravalvular and valvular aortic stenosis in heterozygous familial hypercholesterolemia," Medical Principles and Practice, vol. 16, no. 4, pp. 315-317, 2007.

[21] G. J. Brook, S. Keidar, M. Boulos et al., "Familial homozygous hypercholesterolemia: Clinical and cardiovascular features in 18 patients," Clinical Cardiology, vol. 12, no. 6, pp. 333-338, 1989.

[22] S. Aubert, N. Bonnet, P. Leprince, T. Barreda, A. Pavie, and I. Gandjbakhch, "Surgical approach to aortic root lesions in patients with homozygous familial hypercholesterolemia and Takayasu arteritis," Journal of Thoracic and Cardiovascular Surgery, vol. 130, no. 2, pp. 573-574, 2005.

[23] C. Pitsavos, K. Toutouzas, J. Dernellis et al., "Aortic stiffness in young patients with heterozygous familial hypercholesterolemia," American Heart Journal, vol. 135, no. 4, pp. 604-608, 1998.

[24] B. J. Cairns, S. Coffey, R. C. Travis et al., "A Replicated, GenomeWide Significant Association of Aortic Stenosis With a Genetic Variant for Lipoprotein(a): Meta-Analysis of Published and Novel Data," Circulation, vol. 135, no. 12, pp. 1181-1183, 2017.

[25] R. Vongpromek, S. Bos, G.-J. R. ten Kate et al., "Lipoprotein(a) levels are associated with aortic valve calcification in asymptomatic patients with familial hypercholesterolaemia," Journal of Internal Medicine, vol. 278, no. 2, pp. 166-173, 2015. 


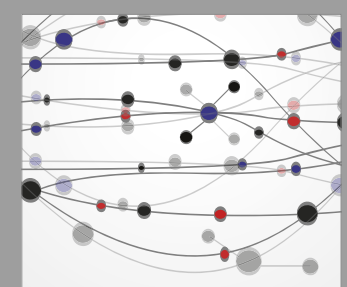

The Scientific World Journal
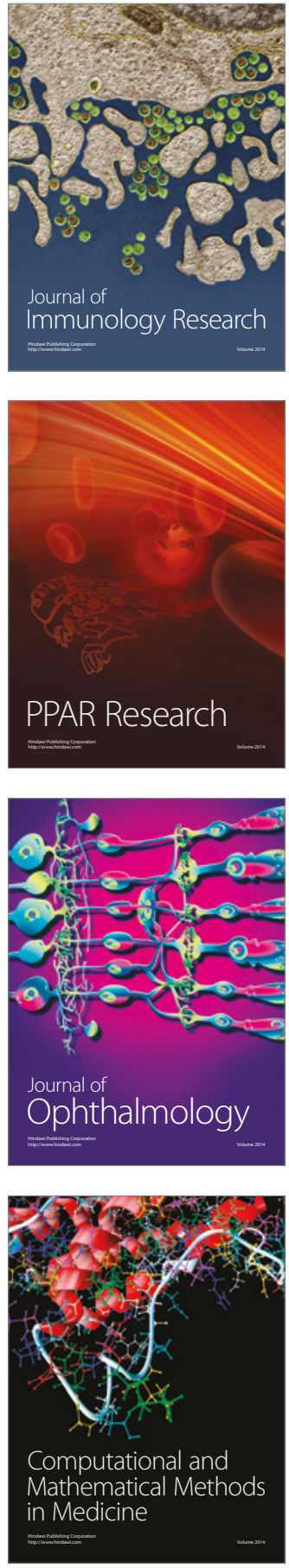

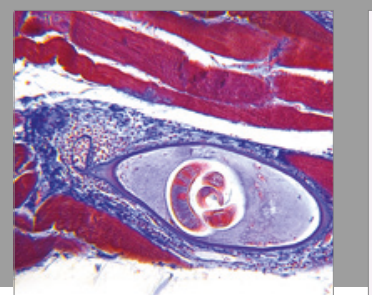

Gastroenterology Research and Practice
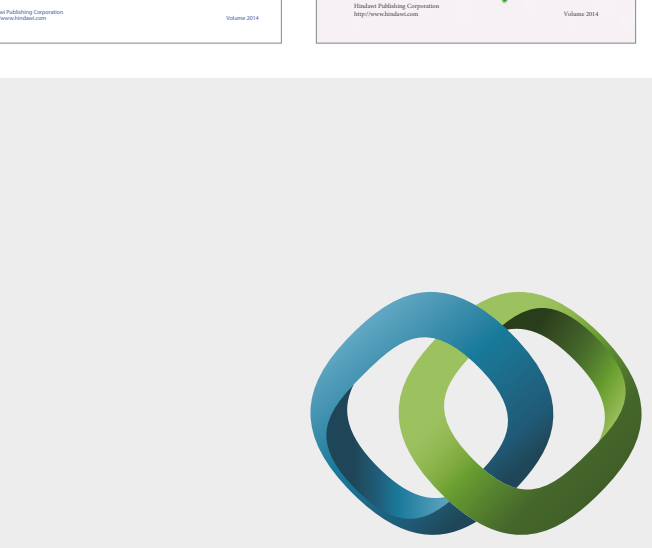

\section{Hindawi}

Submit your manuscripts at

https://www.hindawi.com
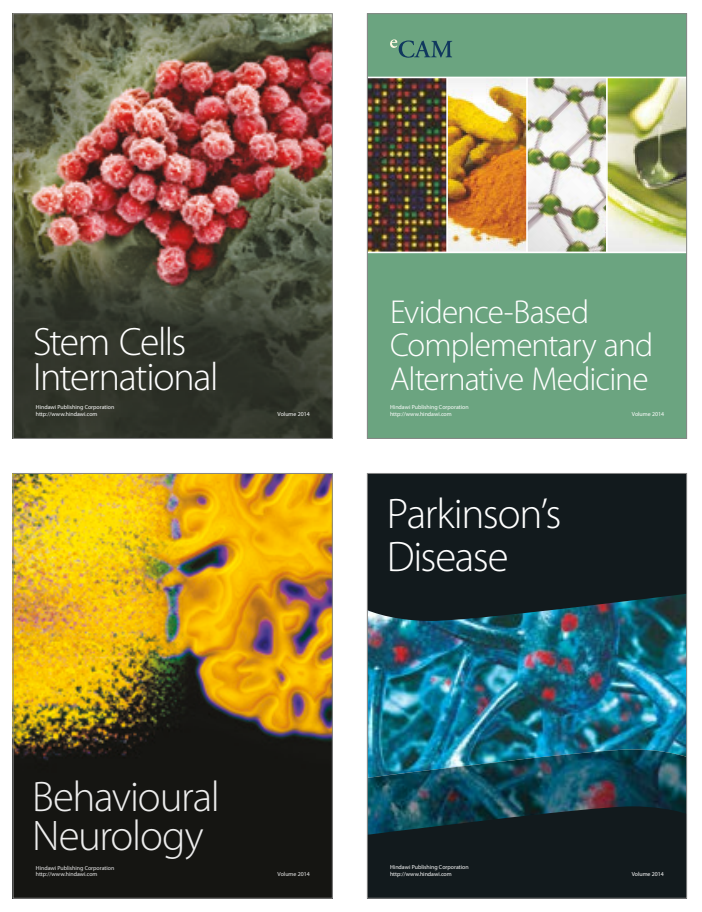
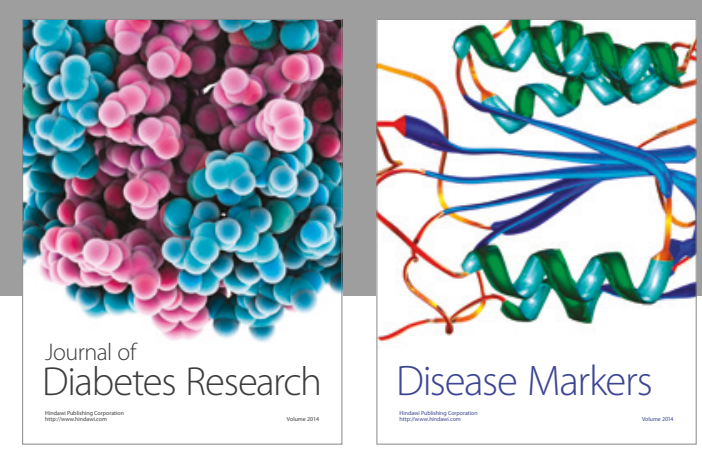

Disease Markers
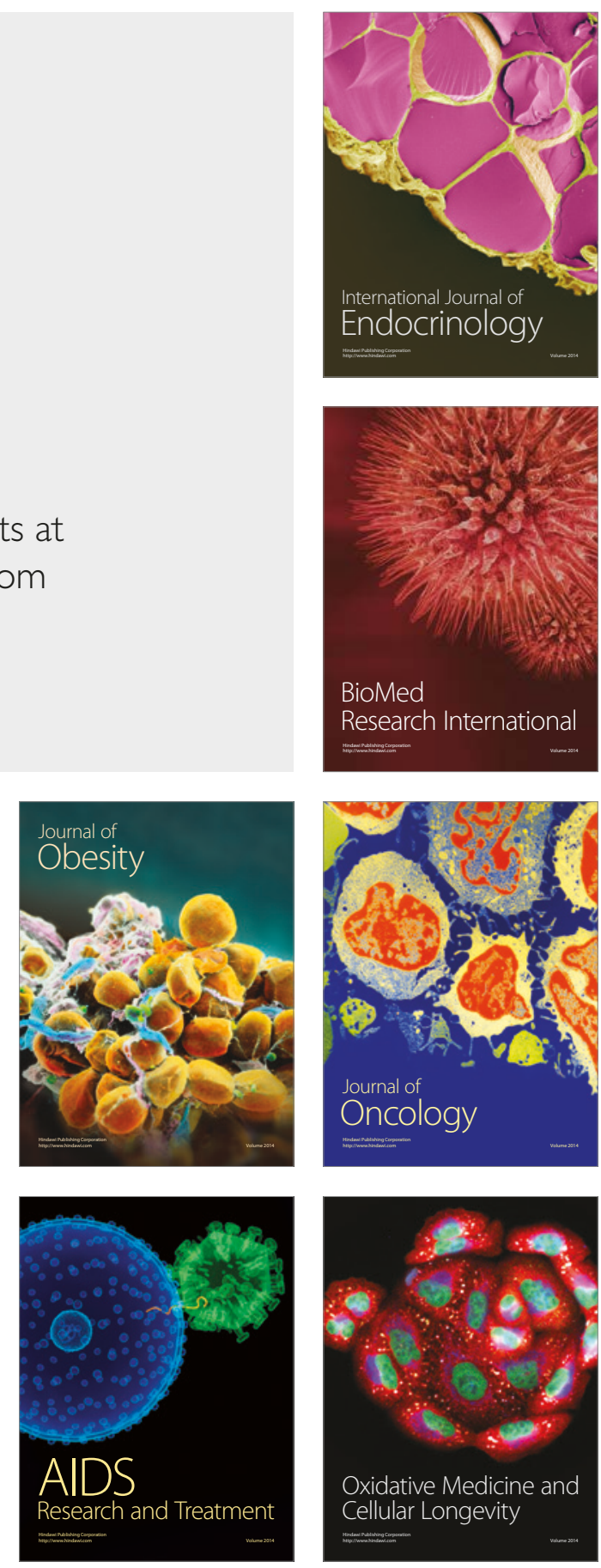